\title{
Dirac R-matrix calculation for electron-impact excitation of S XIII
}

\author{
F. Li $^{1}$, G. Y. Liang ${ }^{1}$, M. A. Bari ${ }^{1,2}$, and G. Zhao ${ }^{1}$ \\ 1 Key Laboratory of Optical Astronomy, National Astronomical Observatories, Chinese Academy of Sciences, 100012 Beijing, \\ PR China \\ e-mail: [gyliang;gzhao]@bao.ac.cn \\ 2 Pakistan Atomic Energy Commission, PO Box 1114, 44000 Islamabad, Pakistan
}

Received 2 May 2013 / Accepted 28 May 2013

\begin{abstract}
Context. Sulfur emission lines in the soft X-ray and extreme-ultraviolet regions are observed in a variety of laboratory and astrophysical spectra. But accurate electron impact excitation data for S XIII for state-of-the-art NLTE spectral models are scarce.

Aims. We calculated electron-impact excitation collision strengths and effective collision strengths of S XIII for transitions among the lowest-lying 98 fine-structure states $1 \mathrm{~s}^{2} 2 \ln l^{\prime}$ corresponding to principal quantum numbers $n=2,3,4$. The effective collision strengths for these transitions were computed over a wide temperature range $\left(\log _{10} T_{\mathrm{e}}(\mathrm{K})=4.53-7.53\right)$ for various astrophysical plasma conditions.

Methods. We used the fully-relativistic parallel Dirac R-matrix code to calculate collision strengths. To generate target wavefunctions and energy levels for scattering calculations, we employed the GRASP0 multi-configuration Dirac-Fock code for states up to $n=5$. Results. The wavefunctions are generated from 27 configurations $-1 \mathrm{~s}^{2} 2 \ln l^{\prime}(n=2,3,4,5)-$ giving rise to $166 j j$ energy levels. The collision and effective collision strengths among the lowest 98 fine-structure levels are compared with the previous theoretical calculations. The collision strengths for most transitions agree well at higher incident electron energies.

Conclusions. The resonant contributions to effective collision strengths are most dominant at lower temperatures.
\end{abstract}

Key words. atomic data - atomic processes - plasmas - line: identification

\section{Introduction}

Sulfur is an abundant element that widely exists in various astrophysical environments (Feldman 1992). In solar and stellar coronae, rich emission lines emanating from sulfur ions have been detected over a wide wavelength range from X-ray to extremeultraviolet (EUV) bands (Acton et al. 1985; Raassen et al. 2002; Li et al. 2013). Be-like ions are of great interest for modelling high-temperature astrophysical and laboratory plasmas, because this isoelectronic sequence provides line ratios suitable for temperature diagnostics (Landi et al. 2001; Kavanagh et al. 2010). The emission lines emanating from S XIII have been used as a test bed to infer electron densities and plasma temperatures (Keenan \& Berrington 1985). The emission lines from S XIII are mainly formed for temperatures in the range of $1.3-16 \mathrm{MK}$, with a peak formation temperature of $2.5 \mathrm{MK}$ (Bryans et al. 2009). Keenan et al. (1988) theoretically investigated the emission line strength of S XIII and compared them with XUV solar flares observations. Moreover, spectral emission lines of S XIII have also been studied extensively in laboratory plasmas. Goldsmith et al. (1974) studied the spectra of S XIII and S XIV and identified twenty new transitions in the 25-40 $\AA$ wavelength range. Khardi et al. (1994) studied the spectra of highly ionised Be-isoelectronic ions (Al, Si, S, and Ar) with a beam-foil experiment in the 50-400 $\AA$ wavelength range. Lepson et al. (2005), with the aid of the Lawrence Livermore electron-beam ion traps EBIT-I and EBIT-II, observed a large number of sulfur emission lines in the soft X-ray and EUV regions. For astrophysical spectra, the authors detected ten strong emission features from S XIII in the 20-75 $\AA$ wavelength range.
Previous calculations about atomic structure data for S XIII have been reported by several authors. Muehlethaler \& Nussbaumer (1976) calculated the transition probabilities for several ions of the Be-isoelectronic sequence within an $n=2$ complex intermediate-coupling scheme. Tully et al. (1990) reported energy levels, oscillator strengths, and photoionisation cross-sections for opacity calculations of fifteen members of the Be-isoelectronic sequence in the Russell Saunders (LS) coupling scheme. Recently, Mania et al. (2009) calculated the excitation energies, lifetimes, and oscillator strengths of levels belonging to the configurations of $1 \mathrm{~s}^{2} 2 \ln l^{\prime}(n=2,3,4,5)$ of S XIII using the multi-configuration Hartree-Fock method, and carried out an extensive comparison with the experimental results. On the experimental side, oscillator strengths, transition rates, and lifetimes within the $n=2$ complex of S XIII have been studied in a number of experimental works (Pegg et al. 1977; Träbert \& Heckmann 1980; Yang et al. 2005; Du et al. 2005; Bhattacharya et al. 1998; Schippers et al. 2012).

Accurate electron-impact excitation data for S XIII are scarse in the literature. The very few previous electron-impact excitation calculations on S XIII have been carried out using nonperturbative Coulomb-Born and distorted wave (DW) methods. An early calculation of collision strengths for 17 Be-like ions $(10 \leq Z \leq 74)$ was performed by Sampson et al. (1984) using the Coulomb-Born-exchange method between levels of $n=2,3$ configurations. Their collision strengths were calculated at nine electron impact energies $(1 \leq \epsilon \leq 15$ in threshold units) in the intermediate-coupling scheme. (Zhang \& Sampson 1992, hereafter ZS92) provided the relativistic DW collision strengths and oscillator strengths for 85 Be-like ions $(8 \leq Z \leq 92)$ for all of the possible $45 \Delta n=0$ transitions among 
10 levels corresponding to $1 \mathrm{~s}^{2} 2 l^{2}$ configurations. This method does not include contributions from resonances. A partial relativistic Coulomb-Bethe (PRCBe) approximation was used to estimate the contributions from large partial waves, which may not be accurate for high incident electron energies (Zhang \& Fontes 2013). Until recently, the most extensive DW collision strength calculation for S XIII was performed by (Landi \& Bhatia 2008, hereafter LB08) using the University College London SUPERSTRUCTURE (SS) code (Eissner et al. 1974). The collision strengths were calculated for lowest eighteen configurations $\left(2 l^{2}, 2 \ln l^{\prime}, n=3,4,5\right.$ with $l=\mathrm{s}, \mathrm{p}$ and $\left.l^{\prime}=\mathrm{s}, \mathrm{p}, \mathrm{d}\right)$ in the Coulomb-Bethe approximation. The authors reported the collision strengths at seven $(10,20,45,90,135,180$, and $225 \mathrm{Ry})$ and five $(45,90,135,180$, and $225 \mathrm{Ry})$ incident electron energies for the transitions within the three lowest $n=2$ configurations and for the transitions between the lowest five levels as well as the levels belonging to the $n=3,4,5$ configurations. This data set is employed in astrophysical spectral modelling packages, such as the current version of the Chianti database (Landi et al. 2012). Resonant contributions to the collision strength in this collisional data set were omitted, which are pertinent for lowtemperature plasmas but their omission is not justified in any accurate electron collisional data. However, higher levels arising from the configurations $2 \mathrm{~s} 4 \mathrm{f}$ and $2 \mathrm{p} 4 \mathrm{f}$ are clearly not included in their calculations, while it is necessary to include them for accurate practical spectral modelling techniques. In addition, the above collision strength calculations focused on a limited number of transitions between levels of $n=2$ configurations and the lowest five levels, corresponding to $n=3,4,5$ configurations.

There are no previous R-matrix calculations for electronimpact excitation data on S XIII apart from the work of (Keenan 1988 , hereafter K88), to the best of our knowledge. In their $\mathrm{R}$-matrix calculation, electron-impact excitation rates for S XIII were calculated by interpolating effective collision strength of three Be-like ions (Ne, Si, Ca) for all transitions amongst $1 \mathrm{~s}^{2} 2 l^{2}$, and results were presented in the form of polynomial fits to the effective collision strengths. Albeit less accurate, it is the only collisional data set on S XIII pertaining to resonance contributions available in the literature to date. In the present work, we primarily intend to perform fully relativistic collision strength calculations for S XIII up to $n=4$ by employing the parallel Dirac R-matrix code (DARC) by taking into account the effect of resonances, since they can lead to a considerable enhancement of the collision rates. All the $98 j j$-levels corresponding to the configurations $1 \mathrm{~s}^{2} 2 l^{2}$ and $1 \mathrm{~s}^{2} 2 \ln l(n=3,4)$ are incorporated to delineate the target structure properly. Then Maxwellianaveraged effective collision strengths are evaluated for electron temperatures $\log _{10} T_{\mathrm{e}}(\mathrm{K})=4.53-7.53$.

\section{Computational methods}

To accomplish collision strength calculations with DARC, we initially need to calculate atomic structure parameters (electronic orbitals, energy levels, and energy states) to delineate the SXIII target ion. In our calculation, we employed the GRASP0 (general-purpose relativistic atomic structure package) code (Grant et al. 1980), which is a fully relativistic $j j$-coupling code based on the multi-configuration Dirac-Fock method. The relativistic corrections arising from the Breit interaction and quantum electrodynamics (QED) effects (vacuum polarisation and Lamb shift) have also been included in GRASP0. For the atomic structure calculations, we optimised the energy levels by using the extended average-level (EAL) mode, in which a weighted (proportional to $2 j+1$ ) trace of the Hamiltonian matrix is minimised. The Lagrange multipliers between orbitals of the same generalised occupation number are set to zero to achieve convergence in a self-consistent field (SCF) process. The wave functions generated by GRASP0 were used as input data for further scattering calculations. The DARC is based on the $j j$ coupling scheme and uses the Dirac-Coulomb Hamiltonian in the R-matrix approach. Relativistic effects were considered in a systematic way in both the target description and the scattering model (colliding electron+target ions). Inclusion of finestructure in the channel coupling increases the size of the corresponding Dirac Hamiltonian matrix substantially. Therefore, it is common practice to perform large-scale calculations in the atomic structure part and then reduce the size of the target state wavefunctions for the scattering part to solve the ion-plus-electron (or $(N+1)$-electron) colliding system.

\subsection{Energy levels}

The wave functions were generated for the 27 lowest-lying configurations $1 \mathrm{~s}^{2} 2 \ln l^{\prime}(n=2,3,4,5)$, which give rise to 166 finestructure levels. The lowest 98 levels in Rydbergs from states up to $n=4$ displayed in Table 1 were employed to construct the target in the scattering calculations. In this table, we also list the experimental results obtained from the Chianti v $7^{1}$ atomic database and the NIST $v 5^{2}$ atomic spectra database to estimate the accuracy of wavefunctions used in the representation of target states. LB08 calculated electron-impact collision strengths, energy levels, oscillator strengths, and transition rates of the 92 fine-structure levels belonging to the configurations $\left(1 \mathrm{~s}^{2} 2 l^{2}, 1 \mathrm{~s}^{2} 2 \ln l^{\prime}\left(n=3,4,5, l^{\prime}=\mathrm{s}, \mathrm{p}, \mathrm{d}\right)\right)$ by using the SS code. Their calculations lack $2 \mathrm{~s} 4 \mathrm{f}$ and $2 \mathrm{p} 4 \mathrm{f}$ configurations, compared with our atomic structure model. Our calculations use the same atomic model as Mania et al. (2009) who employed the multi-configurational Hartree-Fock (MCHF) method.

A comparison of our calculated level energies with those of NIST and other theoretical results is shown in Fig. 1. To provide an immediate overview of the differences between theory and experiment, we plot the ratio of the level energies between the available observed values together with other theoretical results and our calculations. One can see from Fig. 1 and Table 1 that the GRASP0 ten lowest-lying levels $\left(2 l^{2}\right)$ show a considerable discrepancy with NIST values, which is still below $2.5 \%$ however. We differ by $2.2 \%$ at maximum for the $2 \mathrm{~s} 2 \mathrm{p}^{1} \mathrm{P}_{1}$ level from NIST. However, our theoretical model yields more accurate energies of higher excited states, and our results agree within less than $0.2 \%$ for higher configurations with those of NIST. This good agreement between our calculations and the observed values along with the MCDHF/MCHF calculations from the NIST database is consistent for all higher-lying states (see Fig. 1). It is obvious from Fig. 1 that the level energies of our model and the DW energies of LB08 agree within $0.2 \%$. Furthermore, we employed more states originating from two additional configurations (2s4f, 2p4f), than LB08. The addition of these correlation functions improve the accuracy of the target model for the scattering part. Overall, the energy differences seen between our calculations and those of LB08 with the observed energies are very similar. These two sets of calculations agree excellently with each other within $0.2 \%$, as depicted in Fig. 1. The energies of the MCHF calculations of Mania et al. (2009) show a better agreement with the NIST observed values than the LB08 calculations because of energy corrections via a least-squares

\footnotetext{
1 http://www. chiantidatabase.org

http://www.nist.gov/pml/data/asd.cfm
} 
F. Li et al.: Dirac R-matrix calculation for electron-impact excitation of S XIII

Table 1. Level energies (Ryd) of S XIII from different calculations, along with the compilations of experimental values and other theoretical values.

\begin{tabular}{|c|c|c|c|c|c|c|c|c|c|c|c|c|c|c|c|}
\hline ID & Config. & Level & $E_{\mathrm{GRASP}}$ & $E_{\mathrm{SS}}^{a}$ & $E_{\mathrm{MCDHF}}^{b}$ & $E_{\exp }^{d}$ & $\%$ Err & ID & Config. & Level & $E_{\text {GRASP }}$ & $E_{\mathrm{SS}}^{a}$ & $E_{\mathrm{MCDHF}}^{b}$ & $E_{\exp }^{d}$ & $\%$ Err \\
\hline 1 & $2 s^{2}$ & ${ }^{1} \mathrm{~S}_{0}$ & 0.000 & 0.000 & 0.000 & 0.000 & 0.00 & 50 & $2 s 4 p$ & ${ }^{3} \mathrm{P}_{1}$ & 37.022 & 37.028 & $37.028^{c}$ & & \\
\hline 2 & $2 \mathrm{~s} 2 \mathrm{p}$ & ${ }^{3} \mathrm{P}_{0}$ & 1.817 & 1.821 & 1.814 & 1.815 & 0.38 & 51 & $2 \mathrm{~s} 4 \mathrm{p}$ & ${ }^{3} \mathrm{P}_{2}$ & 37.034 & 37.039 & $37.038^{c}$ & & \\
\hline 3 & $2 \mathrm{~s} 2 \mathrm{p}$ & ${ }^{3} \mathrm{P}_{1}$ & 1.860 & 1.862 & 1.853 & 1.854 & 0.43 & 52 & $2 \mathrm{~s} 4 \mathrm{p}$ & ${ }^{1} \mathrm{P}_{1}$ & 37.061 & 37.067 & $37.059^{c}$ & 37.059 & 0.02 \\
\hline 4 & $2 \mathrm{~s} 2 \mathrm{p}$ & ${ }^{3} \mathrm{P}_{2}$ & 1.954 & 1.954 & 1.941 & 1.943 & 0.57 & 53 & $2 \mathrm{~s} 4 \mathrm{~d}$ & ${ }^{3} \mathrm{D}_{1}$ & 37.217 & 37.224 & $37.221^{c}$ & 37.210 & 0.04 \\
\hline 5 & $2 \mathrm{~s} 2 \mathrm{p}$ & ${ }^{1} \mathrm{P}_{1}$ & 3.628 & 3.620 & 3.554 & 3.550 & 1.97 & 54 & $2 \mathrm{~s} 4 \mathrm{~d}$ & ${ }^{3} \mathrm{D}_{2}$ & 37.219 & 37.226 & $37.223^{c}$ & 37.226 & 0.00 \\
\hline 6 & $2 \mathrm{p}^{2}$ & ${ }^{3} \mathrm{P}_{0}$ & 4.803 & 4.807 & 4.770 & 4.768 & 0.82 & 55 & $2 \mathrm{~s} 4 \mathrm{~d}$ & ${ }^{3} \mathrm{D}_{3}$ & 37.222 & 37.229 & $37.225^{c}$ & 37.222 & 0.02 \\
\hline 7 & $2 p^{2}$ & ${ }^{3} \mathrm{P}_{1}$ & 4.856 & 4.860 & 4.819 & 4.819 & 0.85 & 56 & $2 \mathrm{~s} 4 \mathrm{~d}$ & ${ }^{1} \mathrm{D}_{2}$ & 37.335 & 37.340 & $37.313^{c}$ & 37.327 & 0.03 \\
\hline 8 & $2 p^{2}$ & ${ }^{3} \mathrm{P}_{2}$ & 4.938 & 4.937 & 4.894 & 4.892 & 0.92 & 57 & $2 \mathrm{~s} 4 \mathrm{f}$ & ${ }^{3} \mathrm{~F}_{2}$ & 37.341 & & $37.350^{c}$ & & \\
\hline 9 & $2 p^{2}$ & ${ }^{1} \mathrm{D}_{2}$ & 5.456 & 5.458 & 5.376 & 5.371 & 1.62 & 58 & $2 \mathrm{~s} 4 \mathrm{f}$ & ${ }^{3} \mathrm{~F}_{3}$ & 37.342 & & $37.351^{c}$ & & \\
\hline 10 & $2 p^{2}$ & ${ }^{1} \mathrm{~S}_{0}$ & 6.714 & 6.713 & 6.585 & 6.578 & 2.05 & 59 & $2 \mathrm{~s} 4 \mathrm{f}$ & ${ }^{3} \mathrm{~F}_{4}$ & 37.343 & & $37.352^{c}$ & & \\
\hline 11 & $2 \mathrm{~s} 3 \mathrm{~s}$ & ${ }^{3} S_{1}$ & 27.430 & 27.436 & 27.446 & 27.431 & 0.02 & 60 & $2 \mathrm{~s} 4 \mathrm{f}$ & ${ }^{1} \mathrm{~F}_{3}$ & 37.371 & & $37.378^{c}$ & & \\
\hline 12 & $2 \mathrm{~s} 3 \mathrm{~s}$ & ${ }^{1} \mathrm{~S}_{0}$ & 27.775 & 27.780 & 27.785 & & & 61 & $2 p 4 s$ & ${ }^{3} \mathrm{P}_{0}$ & 38.813 & 38.822 & & & \\
\hline 13 & $2 \mathrm{~s} 3 \mathrm{p}$ & ${ }^{1} \mathrm{P}_{1}$ & 28.262 & 28.267 & 28.265 & 28.266 & 0.00 & 62 & $2 \mathrm{p} 4 \mathrm{~s}$ & ${ }^{3} \mathrm{P}_{1}$ & 38.833 & 38.841 & & & \\
\hline 14 & $2 \mathrm{~s} 3 \mathrm{p}$ & ${ }^{3} \mathrm{P}_{0}$ & 28.283 & 28.285 & 28.291 & & & 63 & $2 \mathrm{p} 4 \mathrm{~s}$ & ${ }^{3} \mathrm{P}_{2}$ & 38.956 & 38.957 & & & \\
\hline 15 & $2 \mathrm{~s} 3 \mathrm{p}$ & ${ }^{3} \mathrm{P}_{1}$ & 28.304 & 28.305 & 28.309 & 28.308 & -0.01 & 64 & $2 \mathrm{p} 4 \mathrm{~s}$ & ${ }^{1} \mathrm{P}_{1}$ & 39.022 & 39.022 & & & \\
\hline 16 & $2 \mathrm{~s} 3 \mathrm{p}$ & ${ }^{3} \mathrm{P}_{2}$ & 28.321 & 28.321 & 28.326 & 28.319 & 0.01 & 65 & $2 p 4 p$ & ${ }^{3} \mathrm{D}_{1}$ & 39.052 & 39.061 & $39.153^{c}$ & & \\
\hline 17 & $2 \mathrm{~s} 3 \mathrm{~d}$ & ${ }^{3} \mathrm{D}_{1}$ & 28.770 & 28.772 & 28.772 & 28.776 & -0.14 & 66 & $2 p 4 p$ & ${ }^{3} \mathrm{D}_{2}$ & 39.117 & 39.124 & $39.164^{c}$ & & \\
\hline 18 & $2 \mathrm{~s} 3 \mathrm{~d}$ & ${ }^{3} \mathrm{D}_{2}$ & 28.775 & 28.777 & 28.777 & 28.782 & -0.17 & 67 & $2 p 4 p$ & ${ }^{1} \mathrm{P}_{1}$ & 39.117 & 39.124 & $39.109^{c}$ & & \\
\hline 19 & $2 \mathrm{~s} 3 \mathrm{~d}$ & ${ }^{3} \mathrm{D}_{3}$ & 28.783 & 28.785 & 28.784 & 28.787 & -0.01 & 68 & $2 p 4 p$ & ${ }^{3} \mathrm{P}_{0}$ & 39.188 & 39.193 & $39.175^{c}$ & & \\
\hline 20 & $2 \mathrm{~s} 3 \mathrm{~d}$ & ${ }^{1} \mathrm{D}_{2}$ & 29.127 & 29.129 & 29.101 & 28.380 & 0.10 & 69 & & ${ }^{3} \mathrm{D}_{3}$ & 222 & 39.222 & 39 & & \\
\hline 21 & $2 \mathrm{p} 3 \mathrm{~s}$ & ${ }^{3} \mathrm{P}_{0}$ & 29.722 & 29.730 & 29.725 & & & 70 & $2 p 4 p$ & ${ }^{3} S_{1}$ & 39.227 & 39.228 & & & \\
\hline 22 & $2 \mathrm{p} 3 \mathrm{~s}$ & ${ }^{3} \mathrm{P}_{1}$ & 29.760 & 29.766 & 29.760 & & & 71 & $2 p 4 d$ & ${ }^{3} \mathrm{~F}_{2}$ & 39.251 & 39.259 & $39.258^{c}$ & & \\
\hline 23 & $2 \mathrm{p} 3 \mathrm{~s}$ & ${ }^{3} \mathrm{P}_{2}$ & 29.863 & 29.864 & 29.858 & & & 72 & $2 p 4 p$ & ${ }^{3} \mathrm{P}_{1}$ & 39.283 & 39.283 & $39.234^{c}$ & & \\
\hline 24 & $2 \mathrm{p} 3 \mathrm{~s}$ & ${ }^{1} \mathrm{P}_{1}$ & 30.181 & 30.180 & 30.146 & & & 73 & $2 p 4 p$ & ${ }^{3} \mathrm{P}_{2}$ & 39.288 & 39.287 & & & \\
\hline 25 & $2 p 3 p$ & ${ }^{1} \mathrm{P}_{1}$ & 30.263 & 30.267 & $30.252^{c}$ & 30.249 & 0.06 & 74 & $2 \mathrm{p} 4 \mathrm{~d}$ & ${ }^{3} \mathrm{~F}_{3}$ & 39.302 & 39.309 & $39.302^{c}$ & & \\
\hline 26 & $2 p 3 p$ & ${ }^{3} \mathrm{D}_{1}$ & 30.374 & 30.374 & $30.362^{c}$ & & & 75 & $2 p 4 d$ & ${ }^{1} \mathrm{D}_{2}$ & 39.319 & 39.325 & $39.322^{c}$ & & \\
\hline 27 & $2 p 3 p$ & ${ }^{3} \mathrm{D}_{2}$ & 30.394 & 30.394 & $30.382^{c}$ & 386 & 0.03 & 76 & $2 p 4 d$ & ${ }^{3} \mathrm{D}_{1}$ & 39.366 & 39.373 & $39.373^{c}$ & & \\
\hline 28 & $2 p 3 p$ & ${ }^{3} \mathrm{D}_{3}$ & 30.490 & 30.484 & $30.472^{c}$ & 30.474 & 0.03 & 77 & $2 p 4 p$ & ${ }^{1} \mathrm{D}_{2}$ & 39.370 & 39.370 & $39.353^{c}$ & & \\
\hline 29 & $2 p 3 p$ & ${ }^{3} S_{1}$ & 30.601 & 30.599 & $30.591^{c}$ & 30.579 & 0.07 & 78 & $2 p 4 f$ & ${ }^{3} \mathrm{G}_{3}$ & 39.384 & & $39.495^{c}$ & & \\
\hline 30 & $2 p 3 p$ & ${ }^{3} \mathrm{P}_{0}$ & 30.654 & 30.655 & $30.634^{c}$ & & & 79 & $2 p 4 f$ & ${ }^{3} \mathrm{~F}_{3}$ & 39.394 & & $39.396^{c}$ & & \\
\hline 31 & $2 p 3 p$ & ${ }^{3} \mathrm{P}_{1}$ & 30.710 & 30.708 & $30.680^{c}$ & 30.669 & 0.13 & 80 & $2 p 4 f$ & ${ }^{3} \mathrm{~F}_{4}$ & 39.395 & & $39.397^{c}$ & & \\
\hline 32 & $2 p 3 p$ & ${ }^{3} \mathrm{P}_{2}$ & 30.749 & 30.747 & $30.726^{c}$ & 30.734 & 0.04 & 81 & $2 p 4 d$ & ${ }^{3} \mathrm{~F}_{4}$ & 39.398 & 39.399 & $39.377^{c}$ & & \\
\hline 33 & $2 \mathrm{p} 3 \mathrm{~d}$ & ${ }^{3} \mathrm{~F}_{2}$ & 30.767 & 30.766 & 30.763 & & & 82 & $2 p 4 f$ & ${ }^{3} \mathrm{~F}_{2}$ & 39.401 & & $39.400^{c}$ & & \\
\hline 34 & $2 \mathrm{p} 3 \mathrm{~d}$ & ${ }^{3} \mathrm{~F}_{3}$ & 30.835 & 30.832 & 30.826 & & & 83 & $2 \mathrm{p} 4 \mathrm{~d}$ & ${ }^{3} \mathrm{P}_{2}$ & 39.422 & 39.424 & $39.469^{c}$ & & \\
\hline 35 & $2 \mathrm{p} 3 \mathrm{~d}$ & ${ }^{1} \mathrm{D}_{2}$ & 30.875 & 30.873 & $30.853^{c}$ & & & 84 & $2 p 4 d$ & ${ }^{3} \mathrm{D}_{3}$ & 39.460 & 39.460 & $39.449^{c}$ & 39.462 & -0.01 \\
\hline 36 & $2 \mathrm{p} 3 \mathrm{~d}$ & ${ }^{3} \mathrm{~F}_{4}$ & 30.914 & 30.908 & 30.899 & & & 85 & $2 p 4 d$ & ${ }^{3} \mathrm{D}_{2}$ & 39.490 & 39.490 & $39.410^{c}$ & 39.489 & 0.00 \\
\hline 37 & $2 p 3 p$ & ${ }^{1} \mathrm{D}_{2}$ & 30.990 & 30.991 & $30.946^{c}$ & 30.949 & 0.14 & 86 & $2 p 4 d$ & ${ }^{3} \mathrm{P}_{1}$ & 39.498 & 39.498 & $39.474^{c}$ & & \\
\hline 38 & $2 \mathrm{p} 3 \mathrm{~d}$ & ${ }^{3} \mathrm{D}_{1}$ & 31.079 & 31.083 & 31.078 & 31.046 & 0.12 & 87 & $2 \mathrm{p} 4 \mathrm{~d}$ & ${ }^{3} \mathrm{P}_{0}$ & 39.504 & 39.504 & $39.477^{c}$ & & \\
\hline 39 & $2 \mathrm{p} 3 \mathrm{~d}$ & ${ }^{3} \mathrm{D}_{2}$ & 31.102 & 31.104 & 31.099 & 31.071 & 0.11 & 88 & $2 p 4 f$ & ${ }^{1} \mathrm{~F}_{3}$ & 39.514 & & $39.396^{c}$ & & \\
\hline 40 & $2 \mathrm{p} 3 \mathrm{~d}$ & ${ }^{3} \mathrm{D}_{3}$ & 31.149 & 31.150 & 31.144 & 31.100 & 0.16 & 89 & $2 p 4 f$ & ${ }^{3} \mathrm{G}_{4}$ & 39.521 & & $39.500^{c}$ & & \\
\hline 41 & $2 \mathrm{p} 3 \mathrm{~d}$ & ${ }^{3} \mathrm{P}_{2}$ & 31.233 & 31.230 & 31.222 & 31.170 & 0.19 & 90 & $2 p 4 p$ & ${ }^{1} \mathrm{~S}_{0}$ & 39.524 & 39.523 & $39.540^{c}$ & & \\
\hline 42 & $2 \mathrm{p} 3 \mathrm{~d}$ & ${ }^{3} \mathrm{P}_{1}$ & 31.253 & 31.248 & 31.241 & 31.204 & 0.14 & 91 & $2 p 4 f$ & ${ }^{3} \mathrm{G}_{5}$ & 39.543 & & $39.519^{c}$ & & \\
\hline 43 & $2 \mathrm{p} 3 \mathrm{~d}$ & ${ }^{3} \mathrm{P}_{0}$ & 31.264 & 31.259 & 31.253 & & & 92 & $2 p 4 f$ & ${ }^{3} \mathrm{D}_{3}$ & 39.551 & & $39.527^{c}$ & & \\
\hline 44 & $2 p 3 p$ & ${ }^{1} \mathrm{~S}_{0}$ & 31.417 & 31.417 & $31.331^{c}$ & 31.332 & 0.27 & 93 & $2 p 4 f$ & ${ }^{3} \mathrm{D}_{2}$ & 39.557 & & $39.532^{c}$ & & \\
\hline 45 & $2 \mathrm{p} 3 \mathrm{~d}$ & ${ }^{1} \mathrm{~F}_{3}$ & 31.562 & 31.567 & 31.520 & 31.517 & 0.16 & 94 & $2 p 4 f$ & ${ }^{1} \mathrm{G}_{4}$ & 39.560 & & $39.535^{c}$ & & \\
\hline 46 & $2 \mathrm{p} 3 \mathrm{~d}$ & ${ }^{1} \mathrm{P}_{1}$ & 31.614 & 31.617 & 31.577 & 31.504 & 0.36 & 95 & $2 p 4 f$ & ${ }^{3} \mathrm{D}_{1}$ & 39.583 & & $39.554^{c}$ & & \\
\hline 47 & $2 \mathrm{~s} 4 \mathrm{~s}$ & ${ }^{3} S_{1}$ & 36.687 & 36.696 & $36.676^{c}$ & 36.671 & 0.07 & 96 & $2 p 4 f$ & ${ }^{1} \mathrm{D}_{2}$ & 39.596 & & $39.566^{c}$ & & \\
\hline 48 & $2 \mathrm{~s} 4 \mathrm{~s}$ & ${ }^{1} \mathrm{~S}_{0}$ & 36.813 & 36.818 & $36.797^{c}$ & 36.818 & 0.00 & 97 & $2 \mathrm{p} 4 \mathrm{~d}$ & ${ }^{1} \mathrm{~F}_{3}$ & 39.608 & 39.609 & & 39.489 & \\
\hline 49 & $2 \mathrm{~s} 4 \mathrm{p}$ & ${ }^{3} \mathrm{P}_{0}$ & 37.018 & 37.024 & $37.025^{c}$ & & & 98 & $2 \mathrm{p} 4 \mathrm{~d}$ & ${ }^{1} \mathrm{P}_{1}$ & 39.626 & 39.626 & & & \\
\hline
\end{tabular}

Notes. ${ }^{(a)}$ Landi \& Bhatia (2008); ${ }^{(b)}$ NIST MCDF compilations; ${ }^{(c)}$ Mania et al. (2009); ${ }^{(d)}$ NIST v4 and Chianti v7.

procedure. Such adjustments by Mania et al. (2009) were made to optimise the electronic parameters of the oscillator strengths and lifetimes of S XIII. Their resulting level energies agree excellently within $0.2 \%$ with the observed energies found from the NIST database, as can be seen in Table 1. The agreement between our predicted energies and those of the MCHF calculations by Mania et al. (2009) is also lower than $2.1 \%$ for the lowest-lying ten states, while the energy differences for the higher-lying levels are lower than $0.2 \%$. It should be noted that our atomic model is equal in size to that calculated by Mania et al. (2009). Because of the similarity of these two models, we have listed only a few energy levels in Table 1 from Mania et al. (2009). Table 1 clearly shows that our calculated energies and those of other theoretical calculations agree very well. Based on 


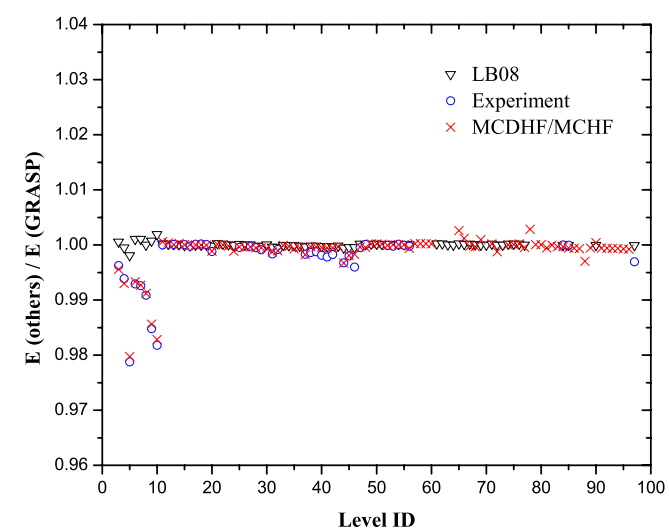

Fig. 1. Ratio between our level energies and previous calculations: black triangle - DW calculations of Landi \& Bhatia (2008), blue circle - experimental values, red cross - both the NIST's MCDHF compilations and MCHF results from Mania et al. (2009).

this analysis, we conclude that there is no (major) discrepancy (better than $2.5 \%$ ) between theory and the experimental values for the energy levels of S XIII. This confirms the reliability of our target used for scattering calculations.

The energy-order of the levels of all three independent calculations (SS/MCHF/GRASP0) is same with a few exceptions. Among the available observed energies, our GRASP0 energyorder differs only in a few cases, namely for levels (45-46, 65-67). Similarly, the level labelling in the SS calculations of LB08 varies for some levels in our GRASP0 and the MCHF calculations of Mania et al. (2009). In Table 1, we present our energy levels by closely following the energy ordering of LB08 to facilitate comparing our results. We expect that energy ordering is accurate due to the small energy differences specifically for strong transitions. Therefore, the overall magnitude of collision strengths is probably not be affected because the level ordering is correct with a few exceptions. It is also helpful to compare the oscillator strengths and $A$-values to better appreciate the quality of wavefunctions used in the target description.

\subsection{Oscillator strengths}

For a given transition $i \rightarrow j$, the absorption oscillator strength $\left(f_{i j}\right)$ and emission transition probabilities $A_{j i}\left(\right.$ in $\mathrm{s}^{-1}$ ) are related by the following expression:

$f_{i j}=\frac{m c}{8 \pi^{2} e^{2}} \lambda_{j i}^{2} \frac{\omega_{j}}{\omega_{i}} A_{i j}=1.49 \times 10^{-16} \lambda_{j i}^{2} \frac{\omega_{j}}{\omega_{i}} A_{j i}$,

where $m$ and $e$ are the electron mass and charge, respectively, $c$ is the velocity of light, $\lambda_{j i}$ is the transition energy/wavelength in $\AA$ and $\omega_{i}$ and $\omega_{j}$ are the statistical weight factors of the initial (i) and final $(j)$ states, respectively.

We computed electric-dipole (E1), magnetic-dipole (M1), electric-quadrupole (E2), and magnetic-quadrupole (M2) transitions amongst the 98 fine-structure levels using the GRASP0 code. In Table 2, a sample of the weighted oscillator strengths ( $g f$-values) and $A$-values of the transitions among the lowest ten levels are compared with the results from the LB08 and MCDHF compilations from the NIST, as well as available experimental $g f$-values. Our $A$-values agree excellently with the results of LB08 and MCDHF compilations.

In Table 2, all transitions obtained from the three sets of calculations agree within $25 \%$, while the agreement for the E1 transitions is better than $15 \%$. Our $g f$-values agree with the experimental values within $10 \%$ expect for a single transition, namely that of $4-8\left(2 \mathrm{~s} 2 \mathrm{p}^{3} \mathrm{P}_{2}-2 \mathrm{p}^{2}{ }^{3} \mathrm{P}_{2}\right)$. Our calculated $g f$-value for this transition shows a maximum discrepancy of $27 \%$ with the corresponding experimental value, but is consistent with other theoretical calculations within $4 \%$. In Fig. 2, we also compare the $g f$-value with the L08 and MCDHF collections from NIST. Only the transitions of the levels up to the $n=3$ configurations are provided in the MCDHF database. Figure 2 illustrates that the oscillator strength of about $89 \%$ of the transitions agree with the L08 calculations within 20\%. Our calculation also agrees with the values from the MCDHF calculations within $20 \%$ for most of the transitions. To conclude, we may state that for almost all strong transitions ( $g f \geq 0.01$ ), Our radiative rates are accurate to better than $20 \%$. However, for some weak transitions $(g f \leq 0.01)$, our calculation differs significantly (by more than a factor of 2) from the results of LB08. Overall, we expect that these atomic structure parameters are reliable because of the small differences with observations. The uncertainties in the collision strength data due to some inaccuracies in the target structure data are correspondingly small.

\subsection{Lifetimes}

The lifetime $\tau$ of an excited level $j$ can be defined as follows:

$\tau_{j}=\frac{1}{\sum_{i} A_{j i}}$.

Lifetimes of excited states can be measured experimentally, and several efforts in the literature have been devoted to measure the lifetimes of excited levels corresponding to the $n=2$ complex of S XIII. To access the accuracy of our calculations, we compared our calculated lifetimes with available experimental values in Table 3. We also derived lifetimes of some levels of S XIII based on the $A$-values of LB08. The lifetimes calculated by Mania adjusted with experimental values are closer to the experimental values than our lifetime predictions with GRASP0. Our lifetimes agree well with the recent experimental measurements (Du et al. 2005) within the measurement uncertainty except for two excited levels $\left(2 \mathrm{~s} 2 \mathrm{p}{ }^{1} \mathrm{P}_{1}, 2 \mathrm{p}^{2}{ }^{3} \mathrm{P}_{0}\right)$, which are lower by $6.25 \%$ and $7.4 \%$, respectively. Our calculated lifetime of the $2 \mathrm{p}^{2}{ }^{3} \mathrm{P}_{0}$ level is $15 \%$ lower than the measured value of Bhattacharya et al. (1998). As for the lifetime for the level $2 \mathrm{~s} 2 \mathrm{p}{ }^{1} \mathrm{P}_{1}$, our calculation is $23 \%$ lower than the measured value of Pegg et al. (1977). Overall, the discrepancy between our calculated lifetimes and all measured ones is within $10 \%$ except for lifetimes of the above two levels. The measured lifetimes agree with each other within the experimental uncertainties. Moreover, theoretical lifetimes are closer to the experimental measurements of $\mathrm{Du}$ et al. (2005), which has the smallest experimental uncertainties among the available measured lifetimes. Based on this comparison, we may state that the accuracy of our calculated lifetimes is better than $10 \%$.

\subsection{Scattering calculations}

The calculations of electron-impact excitation collision strength $(\Omega)$ were performed using a set of parallel DARC R-matrix code ${ }^{3}$ (Ballance \& Griffin 2006), originally developed by Norrington (Norrington \& Grant 1987). The R-matrix boundary radius is about $7.36 \mathrm{au}$, which is determined automatically by the code to enclose most of the target wavefunctions. We employed 30 continuum orbitals for each angular momentum in the expansion of wavefunctions. The allowed energy range

\footnotetext{
http://connorb.freeshell.org/
} 
F. Li et al.: Dirac R-matrix calculation for electron-impact excitation of S XIII

Table 2. Comparison of weighted oscillator strengths and transition rates ( $A$-values) with other theoretical and/or experimental results.

\begin{tabular}{|c|c|c|c|c|c|c|c|c|c|c|}
\hline \multicolumn{2}{|c|}{ Transition } & \multicolumn{3}{|c|}{$g f$-values } & \multicolumn{5}{|c|}{ Transition probability $\left(\mathrm{s}^{-1}\right)$} & \multirow[b]{2}{*}{ Type } \\
\hline$i$ & $j$ & GRASP $^{p}$ & Experiments & $\%$ Err & GRASP $^{p}$ & ${\mathrm{LB} 08^{a}}$ & $\%$ Err & $\mathrm{MCDHF}^{b}$ & $\%$ Err & \\
\hline 1 & 3 & $1.07-04$ & & & $1.00+06$ & $1.12+06$ & 10.30 & $1.10+06$ & 8.67 & E1 \\
\hline 1 & 4 & $7.23-11$ & & & $4.46-01$ & $4.51-01$ & 0.91 & $4.43-01$ & 0.75 & M2 \\
\hline 1 & 5 & $2.33-01$ & $2.29-01^{c}$ & 1.75 & $8.27+09$ & $8.23+09$ & 0.50 & $7.80+09$ & 6.00 & E1 \\
\hline 1 & 7 & $1.31-09$ & & & $8.34+01$ & $1.02+02$ & 17.80 & $8.35+01$ & 0.04 & M1 \\
\hline 1 & 8 & $1.41-09$ & & & $5.57+01$ & $6.19+01$ & 9.91 & $6.02+01$ & 7.38 & $\mathrm{E} 2$ \\
\hline 1 & 9 & $1.21-07$ & & & $5.81+03$ & $5.74+03$ & 1.10 & $5.11+03$ & 13.72 & E1 \\
\hline 2 & 3 & $2.78-07$ & & & $1.39+00$ & $1.62+00$ & 13.94 & $1.42+00$ & 1.61 & M1 \\
\hline 2 & 4 & $3.89-12$ & & & $1.17-04$ & $1.51-04$ & 22.34 & $1.22-04$ & 3.80 & E2 \\
\hline 2 & 5 & $1.89-08$ & & & $1.67+02$ & $1.84+02$ & 9.45 & $1.64+02$ & 1.63 & M1 \\
\hline 2 & 7 & $9.00-02$ & $8.30-02^{c}$ & 8.43 & $2.24+09$ & $2.24+09$ & 0.18 & $2.17+09$ & 3.07 & E1 \\
\hline 2 & 8 & $5.44-11$ & & & $8.57-01$ & $0.00+00$ & & $8.48-01$ & 1.02 & M2 \\
\hline 2 & 9 & $1.13-10$ & & & $2.42+00$ & $0.00+00$ & & $2.20+00$ & 9.81 & M2 \\
\hline 3 & 4 & $8.54-07$ & & & $1.21+01$ & $1.41+01$ & 14.53 & $1.23+01$ & 2.25 & M1, E2 \\
\hline 3 & 5 & $1.45-08$ & & & $1.22+02$ & $1.30+02$ & 5.87 & $1.19+02$ & 2.05 & M1, E2 \\
\hline 3 & 6 & $8.76-02$ & $8.40-02^{c}$ & 4.29 & $6.13+09$ & $6.12+09$ & 0.18 & $5.94+09$ & 3.15 & E1 \\
\hline 3 & 7 & $6.67-02$ & & & $1.61+09$ & $1.61+09$ & 0.00 & $1.56+09$ & 3.15 & $\mathrm{E} 1, \mathrm{M} 2$ \\
\hline 3 & 8 & $1.14-01$ & & & $1.74+09$ & $1.75+09$ & 0.23 & $1.69+09$ & 3.06 & $\mathrm{E} 1, \mathrm{M} 2$ \\
\hline 3 & 9 & $3.09-04$ & & & $6.46+06$ & $7.38+06$ & 12.47 & $8.22+06$ & 21.41 & $\mathrm{E} 1, \mathrm{M} 2$ \\
\hline 3 & 10 & $3.66-05$ & & & $6.98+06$ & $7.49+06$ & 6.80 & $8.33+06$ & 16.26 & E1 \\
\hline 4 & 5 & $2.27-08$ & & & $1.71+02$ & $1.83+02$ & 6.34 & $1.66+02$ & 3.12 & M1, E2 \\
\hline 4 & 6 & $2.93-11$ & & & $1.92+00$ & $0.00+00$ & & $1.83+00$ & 5.11 & M2 \\
\hline 4 & 7 & $1.08-01$ & & & $2.45+09$ & $2.44+09$ & 0.29 & $2.37+09$ & 3.28 & $\mathrm{E} 1, \mathrm{M} 2$ \\
\hline 4 & 8 & $3.27-01$ & $4.45-01^{c}$ & -26.52 & $4.70+09$ & $4.69+09$ & 0.21 & $4.55+09$ & 3.42 & $\mathrm{E} 1, \mathrm{M} 2$ \\
\hline 4 & 9 & $5.51-03$ & & & $1.09+08$ & $1.20+08$ & 8.70 & $1.22+08$ & 10.58 & $\mathrm{E} 1, \mathrm{M} 2$ \\
\hline 4 & 10 & $1.15-10$ & & & $2.11+01$ & $0.00+00$ & & $1.94+01$ & 9.18 & M2 \\
\hline 5 & 6 & $1.08-04$ & & & $1.21+06$ & $1.40+06$ & 13.56 & $1.48+06$ & 18.01 & E1 \\
\hline 5 & 7 & $3.08-05$ & & & $1.25+05$ & $1.43+05$ & 12.83 & $1.50+05$ & 16.39 & E1, M2 \\
\hline 5 & 8 & $1.83-03$ & & & $5.08+06$ & $5.80+06$ & 12.35 & $6.63+06$ & 23.34 & $\mathrm{E} 1, \mathrm{M} 2$ \\
\hline 5 & 9 & $2.58-01$ & $2.73-01^{d}$ & -5.49 & $1.39+09$ & $1.42+09$ & 1.97 & $1.38+09$ & 1.07 & $\mathrm{E} 1, \mathrm{M} 2$ \\
\hline 5 & 10 & $1.68-01$ & $1.71-01^{d}$ & -1.75 & $1.29+10$ & $1.29+10$ & 0.23 & $1.20+10$ & 7.72 & E1 \\
\hline 6 & 7 & $3.91-07$ & & & $2.93+00$ & $3.47+00$ & 15.52 & $2.86+00$ & 2.30 & M1 \\
\hline 6 & 8 & $3.39-12$ & & & 9.91-05 & $1.26-04$ & 21.37 & $1.01-04$ & 1.71 & E2 \\
\hline 6 & 9 & $8.20-15$ & & & $5.65-06$ & 5.99-06 & 5.63 & $1.60-05$ & 64.70 & $\mathrm{E} 2$ \\
\hline 7 & 8 & $6.44-07$ & & & $6.97+00$ & $7.94+00$ & 12.26 & $7.13+00$ & 2.27 & M1, E2 \\
\hline 7 & 9 & $8.78-08$ & & & $5.11+01$ & $5.83+01$ & 12.31 & $5.00+01$ & 2.18 & M1, E2 \\
\hline 7 & 10 & $6.22-08$ & & & $1.74+03$ & $1.90+03$ & 8.64 & $1.68+03$ & 3.22 & M1 \\
\hline 8 & 9 & $2.37-07$ & & & $1.03+02$ & $1.14+02$ & 9.64 & $9.72+01$ & 6.05 & M1, E2 \\
\hline 8 & 10 & $3.25-10$ & & & $8.29+00$ & $8.97+00$ & 7.54 & $7.64+00$ & 8.48 & E2 \\
\hline 9 & 10 & $1.62-08$ & & & $2.07+02$ & $1.98+02$ & 4.91 & $1.65+02$ & 25.99 & E2 \\
\hline
\end{tabular}

Notes. ${ }^{(p)}$ This work; ${ }^{(a)}$ Landi \& Bhatia (2008); ${ }^{(b)}$ NIST MCDHF values; ${ }^{(c)}$ Yang et al. (2005); ${ }^{(d)}$ Pegg et al. (1977) .

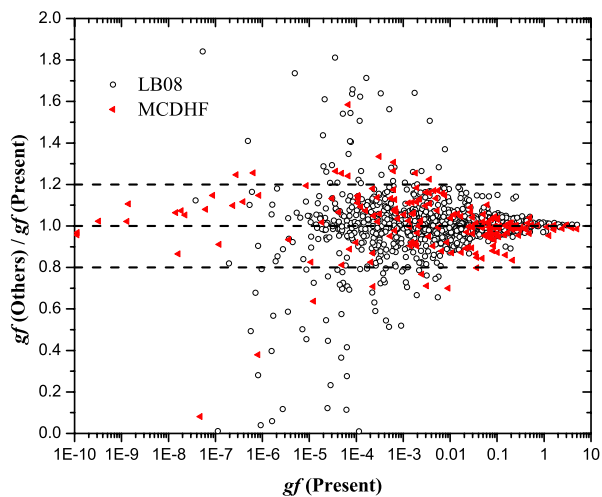

Fig. 2. Ratio between our $g f$-values and other theoretical calculations: open circle - DW calculations of Landi \& Bhatia (2008), solid triangle - NIST's MCDHF collections.

for $\Omega$ can be up to 160 Ryd, which is about three times the ionisation potential of S XIII. Contributions from partial waves up to $J=41.5$ are included. This is sufficient for most transitions in the energy range $\lesssim 160$ Ryd. However, a large number of the $J$-value would be preferred for some allowed transitions above the resonance region. To account for the contribution of higher partial waves, we included a "top-up", based on the Coulomb-Bethe method (Burgess \& Sheorey 1974) in our calculation. In the resonance region, we used a fine energy mesh of $8.317 \times 10^{-6} z^{2}$ Ryd and $10^{4}$ energy points (up to $47.9 \mathrm{Ryd}$ ), where $z$ is the effective or residual charge (12) of the ion. This is sufficient to resolve the resonance structures in the closed channel region. Above 47.9 Ryd and up to three times the ionisation potential of this ion, a coarser energy mesh $\left(6.655 \times 10^{-4} z^{2}\right.$ Ryd $)$ and 2000 energy points were employed.

In astrophysical and laboratory plasma applications, often the excitation rate coefficients (in units of $\mathrm{cm}^{3} \mathrm{~s}^{-1}$ ) are used. In this context, the excitation rate coefficient for a transition $i \rightarrow j$ can be expressed as follows:

$C_{i j}=\frac{8.63 \times 10^{-6}}{g_{i} T_{\mathrm{e}}^{1 / 2}} \mathrm{e}^{-\frac{\Delta E_{i j}}{k T_{\mathrm{e}}}} \Upsilon_{i j}$, 
Table 3. Comparison of the lifetimes with available experimental results.

\begin{tabular}{|c|c|c|c|c|c|c|c|c|c|}
\hline \multicolumn{2}{|c|}{ Transition } & \multicolumn{2}{|c|}{ Wavelength $(\AA)$} & \multicolumn{6}{|c|}{ Lifetime of the upper level (ps) } \\
\hline Lower level & Upper level & This work & Observed $^{a}$ & This work & ML09 $^{b}$ & LB08 $^{c}$ & PF77 $^{d}$ & $\mathrm{BB} 98^{e}$ & $\mathrm{DB} 05^{f}$ \\
\hline $2 \mathrm{~s}^{2}\left({ }^{1} \mathrm{~S}_{0}\right)$ & $2 \mathrm{~s} 2 \mathrm{p}\left({ }^{1} \mathrm{P}_{1}\right)$ & 251.18 & 256.89 & 120.95 & 124.3 & 121.55 & $157 \pm 16$ & $129 \pm 6$ & $129 \pm 4$ \\
\hline $2 \mathrm{~s} 2 \mathrm{p}\left({ }^{1} \mathrm{P}_{1}\right)$ & $2 \mathrm{p}^{2}\left({ }^{1} \mathrm{~S}_{0}\right)$ & 295.23 & 301.18 & 77.42 & 85.28 & 77.59 & $79 \pm 15$ & $84 \pm 10$ & $81 \pm 5$ \\
\hline $2 \mathrm{~s} 2 \mathrm{p}\left({ }^{1} \mathrm{P}_{1}\right)$ & $2 \mathrm{p}^{2}\left({ }^{1} \mathrm{D}_{2}\right)$ & 498.35 & 500.81 & 663.28 & 657.4 & 646.42 & $689 \pm 35$ & $740 \pm 45$ & \\
\hline $2 \mathrm{~s} 2 \mathrm{p}\left({ }^{3} \mathrm{P}_{2}\right)$ & $2 \mathrm{p}^{2}\left({ }^{3} \mathrm{P}_{2}\right)$ & 305.39 & 309.25 & & & & & & \\
\hline $2 \mathrm{~s} 2 \mathrm{p}\left({ }^{3} \mathrm{P}_{1}\right)$ & $2 \mathrm{p}^{2}\left({ }^{3} \mathrm{P}_{2}\right)$ & 296.11 & 300.19 & 155.06 & 158.1 & 155.19 & $168 \pm 9$ & $173 \pm 20$ & $159 \pm 8$ \\
\hline $2 \mathrm{~s} 2 \mathrm{p}\left({ }^{3} \mathrm{P}_{0}\right)$ & $2 \mathrm{p}^{2}\left({ }^{3} \mathrm{P}_{1}\right)$ & 299.87 & 303.59 & & & & & & \\
\hline $2 \mathrm{~s} 2 \mathrm{p}\left({ }^{3} \mathrm{P}_{1}\right)$ & $2 \mathrm{p}^{2}\left({ }^{3} \mathrm{P}_{1}\right)$ & 304.20 & 307.58 & & & & & & \\
\hline $2 \mathrm{~s} 2 \mathrm{p}\left({ }^{3} \mathrm{P}_{2}\right)$ & $2 \mathrm{p}^{2}\left({ }^{3} \mathrm{P}_{1}\right)$ & 319.80 & 317.10 & 158.70 & 161.7 & 158.78 & & $177 \pm 9$ & $165 \pm 7$ \\
\hline $2 \mathrm{~s} 2 \mathrm{p}\left({ }^{3} \mathrm{P}_{1}\right)$ & $2 \mathrm{p}^{2}\left({ }^{3} \mathrm{P}_{0}\right)$ & 309.64 & 312.96 & 163.05 & 165.9 & 163.33 & & $192 \pm 15$ & $176 \pm 11$ \\
\hline
\end{tabular}

Notes. ${ }^{(a)}$ Calculated from the observed level energies in Table 1; ${ }^{(b)}$ calculated from the A-values of Landi \& Bhatia (2008); ${ }^{(c)}$ Mania et al. (2009); ${ }^{(d)}$ Pegg et al. (1977); ${ }^{(e)}$ Bhattacharya et al. (1998); ${ }^{(f)}$ Du et al. (2005).

where $T_{\mathrm{e}}$ is the electron temperature in $\mathrm{K}, \Delta E_{i j}$ is the transition energy, and $k$ is the Boltzmann constant, the $\Upsilon_{i j}$ is the the dimensionless thermally averaged effective collision strength, which can be defined as follows:

$\Upsilon_{i j}=\int_{0}^{+\infty} \Omega_{i j}\left(E_{i j}\right) \mathrm{e}^{-\frac{E_{j}}{k T_{\mathrm{e}}}} \mathrm{d}\left(\frac{E_{j}}{k T_{\mathrm{e}}}\right)$,

where $E_{j}$ is the incident energy with respect to the level $j$. The effective collision strengths were calculated in a wide temperature range $\left(3.38 \times 10^{4}-3.38 \times 10^{7} \mathrm{~K}\right)$ to encompass all temperatures of importance in astrophysical and plasma applications. The high-energy Born/Bethe limits were calculated using the AUTOSTRUCTURE (AS) code (Badnell 1986). These limits were then used to extrapolate the collision strength at the energy above 160 Ryd to obtain the effective collision strength at high temperatures.

\section{Results and discussions}

Collision strengths and the Maxwellian-averaged effective collision strengths among 98 fine-structure levels of S XIII for a total of 4753 transitions were calculated adopting a suite of parallel Dirac R-matrix programs. Therefore, it is not feasible to tabulate the collision strengths and the effective collision strengths because of the large number of transitions and the incident electron energies involved. A complete set of collision strengths and effective collision strengths for all transitions involved can be obtained online. In this section, we solely focus on the collision strength and the corresponding effective collision strength of some typical transitions. Figures 3-7 display the collision strengths and/or the associated effective collision strengths for the selected transitions. Comparisons of these electron-impact collisional parameters are mainly made from the earlier R-matrix work of K88 and the two independent DW calculations by ZS92 and LB08. The selection of transitions shown in Figs. 3-7 are based on the kinds of agreement and the extent of differences we observe with previous theoretical calculations. Here, we also present Table 4 to compare a sample of the current effective collision strengths with those from the R-matrix calculations of K88 and the DW calculation of LB08. Only the values at three temperatures $\left(\log _{10} T_{\mathrm{e}}(\mathrm{K})=6.23,6.53,6.93\right)$ are displayed in Table 4.

In Fig. 3, we compare the collision strength for the optically allowed transition $1-5\left(2 \mathrm{~s}^{2}{ }^{1} \mathrm{~S}_{0}-2 \mathrm{~s} 2 \mathrm{p}{ }^{1} \mathrm{P}_{1}\right)$ with the available DW collision strengths of LB08, who reported the collision strengths of S XIII at seven and five incident electron energies,

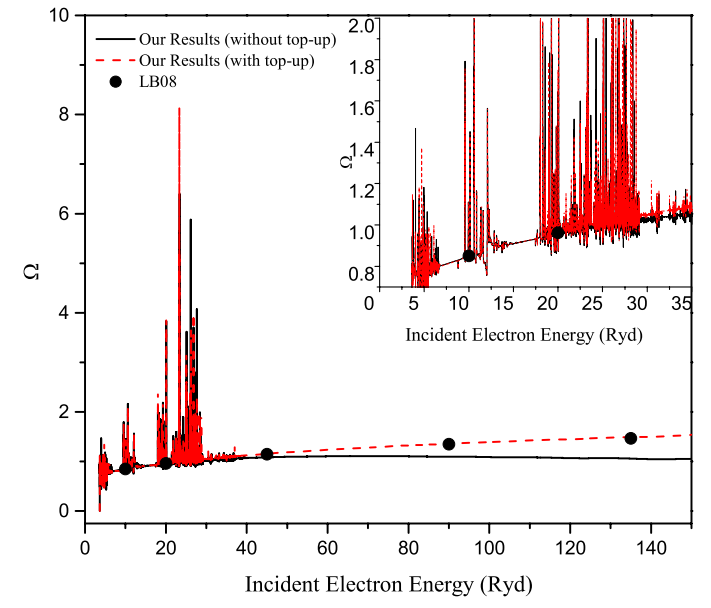

Fig. 3. Collision strength for the $1-5\left(2 \mathrm{~s}^{2}{ }^{1} \mathrm{~S}_{0}-2 \mathrm{~s} 2 \mathrm{p}{ }^{1} \mathrm{P}_{1}\right)$ transition: solid black curve - our calculation without "top-up", dashed red curve - our calculation with "top-up", solid circle - DW calculations of Landi \& Bhatia (2008).

as described above. In Fig. 3, our results with "top-up" procedure are shown with the dashed red curve, while the solid black curve displays our results without the "top-up" method. The background values of the collision strength calculated with the "top-up" procedure agree well with LB08, but the collision strength without the "top-up" is lower than LB08 by $27 \%$, especially above the threshold excitation. From Fig. 3, we can see that if we neglect this "top-up" procedure to our collision strength calculations, it will lead to inaccuracies in the calculated results for higher partial waves, which ultimately will affect the calculations of the effective collision strengths. Furthermore, we see from the inset in Fig. 3 that the "top-up" contributions near the resonance threshold to the collision strengths of the dipole-allowed transition are negligible and the DW calculations agree well with the background level in this low-energy resonance region. We also compared all available transitions reported in LB08 and ZS92. Our results for the background of the collision strength agree well with LB08 and ZS92 for the vast majority of transitions with a few exceptions. For example, LB08 noticed that their collision strengths for the transition $1-9\left(2 \mathrm{~s}^{2}{ }^{1} \mathrm{~S}_{0}-2 \mathrm{p}^{2}{ }^{1} \mathrm{D}_{2}\right)$ and $1-10\left(2 \mathrm{~s}^{2}{ }^{1} \mathrm{~S}_{0}-2 \mathrm{p}^{2}{ }^{1} \mathrm{~S}_{0}\right)$ are higher by a factor of 1.5-2.0 than the results of ZS92. For the transition 1-9, the background level of our collision strength is slightly lower than LB08, but higher than ZS92. This difference is caused by the configuration interaction effects, which have 
F. Li et al.: Dirac R-matrix calculation for electron-impact excitation of S XIII
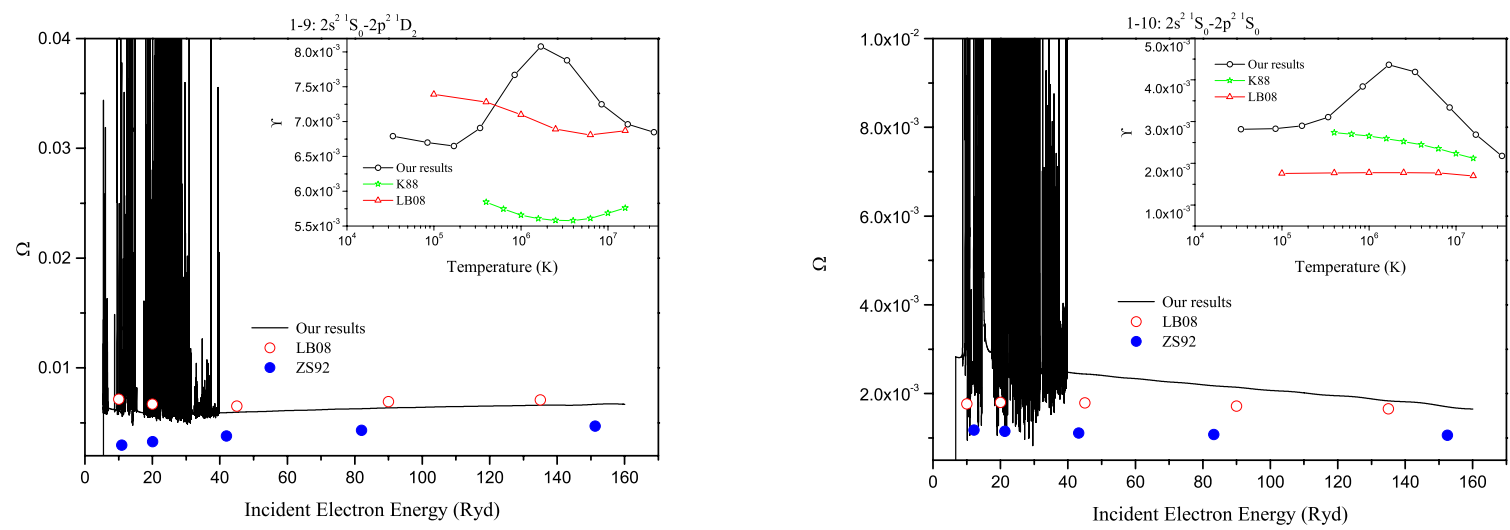

Fig. 4. Collision strength 1-9 and 1-10: solid black curve - our DARC results, solid blue circle - DW calculation of Zhang \& Sampson (1992), open red circle - DW calculation of Landi \& Bhatia (2008). The insets in the graphs compare the corresponding effective collision strength ( $\Upsilon$ ) as a function of the electron temperature: open black circle - our DARC results, open star - R-matrix interpolated work of Keenan (1988), open upward triangle - DW calculations from Landi \& Bhatia (2008).
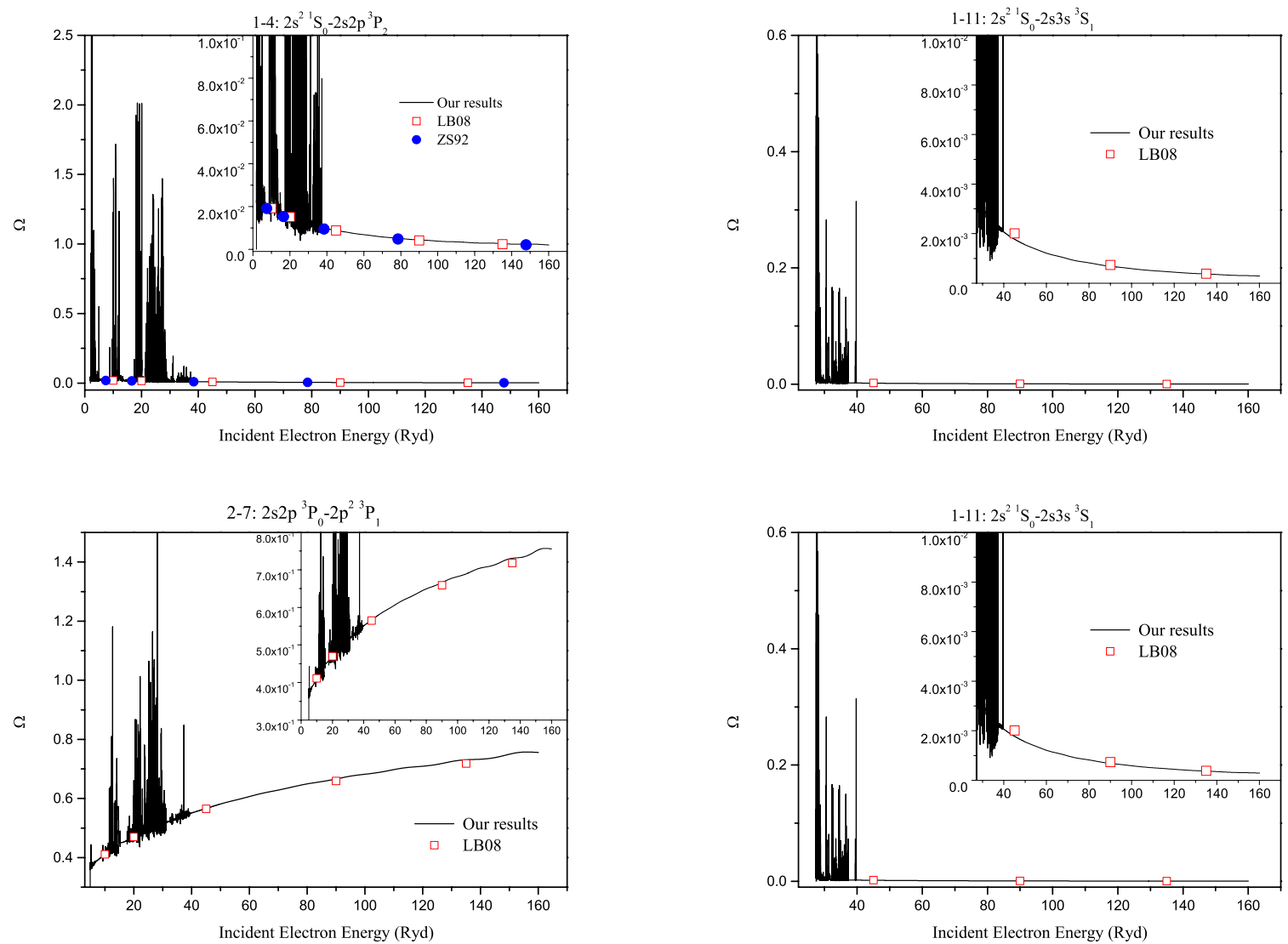

Fig. 5. Collision strengths for the optically allowed transitions $1-13$ and 2-7, optically forbidden transition 1-4, and the intercombination transition 1-11: solid curve - our DARC calculation, open red square - DW calculations from Landi \& Bhatia (2008), solid blue circle - calculations from Zhang \& Sampson (1992). The insets show the details of the collision strengths near the background.

been explored in LB08. It is obvious from Fig. 4 that the background level of our collision strength for the transition 1-10 is higher by a factor of 1.5-2.0 than those of LB08 in the threshold region, but converges in the high-energy region. The effect of differing collisions between our results and the LB08 calculations on the resulting effective collision strengths are shown in the insets in Fig. 4. These two insets clearly show the impact of resonances on the effective collision strength for both transitions. The available effective collision strength data for the two transitions from the interpolated work of K88 and the DW calculations of LB08 over a range of temperature exhibit strong deviations from our results. There is even little or no agreement for all three independent calculations for the 1-9 transition with the exception of a single temperature value of LB08 in the highertemperature domain, where the background level of our collision strength and that of LB08 agree well. For the low-temperature region, the height of the low-lying resonances enhances the effective collision strength significantly. For the 1-10 transition, 

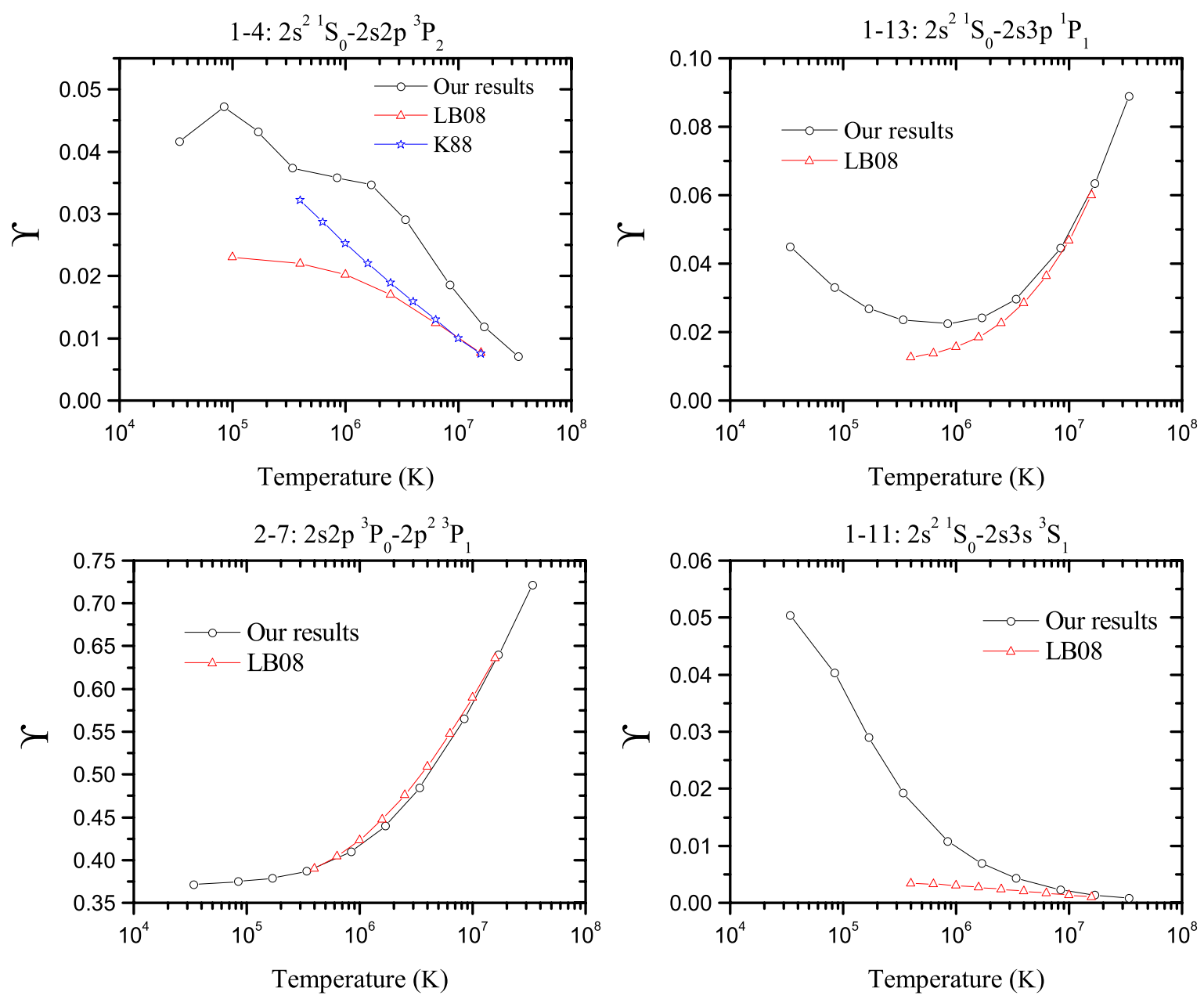

Fig. 6. Comparison of our DARC effective collision strengths: open circle - our calculation, open red upward triangle - DW calculation of Landi \& Bhatia (2008), open blue star - interpolated R-matrix values of Keenan (1988).

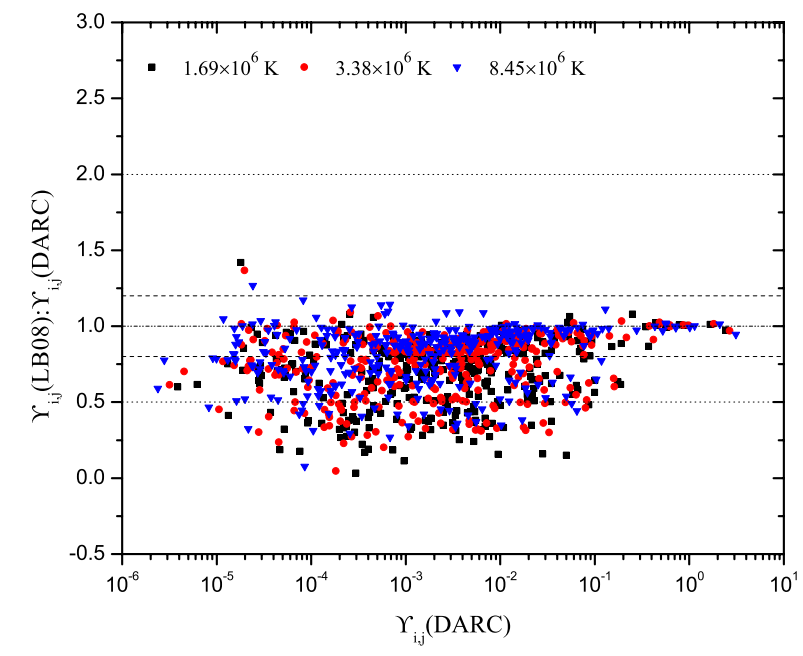

Fig. 7. Comparison of our effective collision strengths with those of Landi \& Bhatia (2008): dashed lines indicate agreement within 20\%, dotted lines indicate agreement within a factor of 2 .

the effective collision strengths produced by K88 are approximately 3\%-78\% lower, while the LB08 predictions $61 \%-78 \%$ are lower than our DARC values over the temperature range. Based on this analysis, both of these earlier calculations are not sophisticated enough because they exclude resonances and produce an underestimated effective collision strength for the two transitions. The same trend is observed for the two calculations with our R-matrix results for the 1-10 transition. The K88 results are employed by the Chianti database because they include the resonance effects. However, as can be seen in the inset panel of Fig. 4, the K88's effective collision strength for the transitions 1-9 is lower than our results by about $30 \%$ and lower than the LB08 results. For most transitions, the effective collision strengths of K88 are lower than our calculation, but higher than that of LB08, which clearly indicates that the resonance contributions are underestimated.

In Fig. 5, we consider the collision strengths for the optically forbidden transition $1-4\left(2 \mathrm{~s}^{2}{ }^{1} \mathrm{~S}_{0}-2 \mathrm{~s} 2 \mathrm{p}{ }^{3} \mathrm{P}_{2}\right)$, the spin-exchange transition $1-11\left(2 \mathrm{~s}^{2}{ }^{1} \mathrm{~S}_{0}-2 \mathrm{~s} 3 \mathrm{~s}^{3} \mathrm{~S}_{1}\right)$, and the two optically allowed transitions 1-13 $\left(2 \mathrm{~s}^{2}{ }^{1} \mathrm{~S}_{0}-2 \mathrm{~s} 3 \mathrm{p}^{1} \mathrm{P}_{1}\right)$ and 2-7 (2s $\left.2 \mathrm{p}^{3} \mathrm{P}_{0}-2 \mathrm{p}^{2}{ }^{3} \mathrm{P}_{1}\right)$. Here, we again compare our collision strength values for the four transitions with the two previous DW calculations of ZS92 and LB08. It is clear from Fig. 5 that our background collision strengths for all four transitions agree well with them. The insets in Fig. 5 show that the resonance effects dominate the collision strength in the low-energy region and are larger than the background level of the collision strength in the high-energy region. Our results agree excellently with the previous DW results for all transitions with a few exceptions. The resonant effects can be up to 2-3 orders of magnitude except for transition 2-7, which is only a few times higher than the background. 
F. Li et al.: Dirac R-matrix calculation for electron-impact excitation of S XIII

Table 4. Effective collision strength for the first 10 levels in S XIII at three temperatures $\left(\log _{10} T_{\mathrm{e}}(\mathrm{K})=6.23,6.53\right.$, and 6.93$)$.

\begin{tabular}{|c|c|c|c|c|c|c|c|c|c|c|}
\hline \multicolumn{2}{|c|}{ Transition } & \multicolumn{3}{|c|}{6.23} & \multicolumn{3}{|c|}{6.53} & \multicolumn{3}{|c|}{6.93} \\
\hline $\mathrm{i}$ & $\mathrm{j}$ & $\mathrm{DARC}^{a}$ & $\mathrm{~K} 88^{b}$ & ${\mathrm{LB} 08^{c}}^{c}$ & $\mathrm{DARC}^{a}$ & $\mathrm{~K} 88^{b}$ & ${\mathrm{LB} 08^{c}}^{c}$ & $\mathrm{DARC}^{a}$ & $\mathrm{~K} 88^{b}$ & $\mathrm{LB}^{\circ}{ }^{c}$ \\
\hline 1 & 2 & $7.08-03$ & $4.30-03$ & $1.28-03$ & $5.83-03$ & $3.38-03$ & $7.60-04$ & $3.70-03$ & $2.23-03$ & $3.36-04$ \\
\hline 1 & 3 & $2.17-02$ & $1.40-02$ & $5.57-03$ & $1.85-02$ & $1.14-02$ & $4.37-03$ & $1.25-02$ & 8.06-03 & $3.57-03$ \\
\hline 1 & 4 & $3.47-02$ & $2.16-02$ & $6.33-03$ & $2.91-02$ & $1.69-02$ & $3.76-03$ & $1.86-02$ & $1.10-02$ & $1.66-03$ \\
\hline 1 & 5 & 8.94-01 & $9.00-01$ & $1.35+00$ & $9.82-01$ & $9.89-01$ & $1.52+00$ & $1.15+00$ & $1.14+00$ & $1.74+00$ \\
\hline 1 & 6 & $4.10-04$ & $1.36-04$ & $5.83-05$ & $3.92-04$ & $1.11-04$ & $3.88-05$ & $2.57-04$ & $7.59-05$ & $2.37-05$ \\
\hline 1 & 7 & $1.12-03$ & $3.59-04$ & $1.32-04$ & $1.06-03$ & $2.89-04$ & $7.56-05$ & $6.80-04$ & $1.83-04$ & $3.29-05$ \\
\hline 1 & 8 & $1.74-03$ & $6.53-04$ & $3.37-04$ & $1.62-03$ & $5.30-04$ & $2.49-04$ & $1.07-03$ & $3.67-04$ & $1.83-04$ \\
\hline 1 & 9 & 8.08-03 & $5.60-03$ & $6.95-03$ & $7.88-03$ & $5.58-03$ & $7.00-03$ & $7.25-03$ & $5.65-03$ & $7.02-03$ \\
\hline 1 & 10 & $4.36-03$ & $2.58-03$ & $1.67-03$ & 4.19-03 & $2.48-03$ & $1.59-03$ & $3.34-03$ & $2.28-03$ & $1.48-03$ \\
\hline 2 & 3 & $7.46-02$ & $7.32-02$ & $1.23-02$ & $5.68-02$ & 5.11-02 & $7.22-03$ & $3.45-02$ & $3.06-02$ & $3.17-03$ \\
\hline 2 & 4 & $5.41-02$ & $5.75-02$ & $2.40-02$ & $4.58-02$ & $4.20-02$ & $2.38-02$ & $3.51-02$ & $3.14-02$ & $2.38-02$ \\
\hline 2 & 5 & $1.92-02$ & $1.06-02$ & $2.80-03$ & $1.62-02$ & $8.07-03$ & $1.62-03$ & $9.98-03$ & $5.19-03$ & 7.09-04 \\
\hline 2 & 6 & $3.63-03$ & $2.55-03$ & $7.93-04$ & $3.15-03$ & $2.08-03$ & 4.69-04 & $2.07-03$ & $1.41-03$ & $2.07-04$ \\
\hline 2 & 7 & $4.40-01$ & $4.51-01$ & $6.60-01$ & $4.84-01$ & $4.97-01$ & $7.42-01$ & $5.65-01$ & $5.74-01$ & 8.44-01 \\
\hline 2 & 8 & $8.18-03$ & $2.95-03$ & $1.34-03$ & 7.17-03 & $2.61-03$ & 7.93-04 & $4.58-03$ & $1.90-03$ & $3.50-04$ \\
\hline 2 & 9 & $1.00-02$ & $4.79-03$ & $1.68-03$ & $8.74-03$ & $4.01-03$ & $9.90-04$ & $5.54-03$ & $2.72-03$ & 4.36-04 \\
\hline 2 & 10 & $1.61-03$ & $6.10-04$ & $1.79-04$ & $1.36-03$ & $5.09-04$ & $1.04-04$ & $8.22-04$ & $3.46-04$ & $4.56-05$ \\
\hline 3 & 4 & $2.50-01$ & $2.70-01$ & $6.89-02$ & $1.92-01$ & $1.98-01$ & $6.26-02$ & $1.30-01$ & $1.45-01$ & $5.79-02$ \\
\hline 3 & 5 & $5.84-02$ & $3.22-02$ & $8.55-03$ & $4.89-02$ & $2.44-02$ & $5.17-03$ & $3.02-02$ & $1.60-02$ & $2.55-03$ \\
\hline 3 & 6 & $4.44-01$ & $4.46-01$ & $6.67-01$ & $4.88-01$ & $4.95-01$ & 7.49-01 & $5.71-01$ & $5.75-01$ & $8.53-01$ \\
\hline 3 & 7 & $3.47-01$ & $3.46-01$ & 4.99-01 & $3.78-01$ & $3.79-01$ & $5.60-01$ & $4.34-01$ & $4.33-01$ & $6.36-01$ \\
\hline 3 & 8 & $5.66-01$ & $5.70-01$ & $8.25-01$ & 6.19-01 & $6.24-01$ & $9.27-01$ & 7.15-01 & 7.14-01 & $1.05+00$ \\
\hline 3 & 9 & $3.37-02$ & $1.70-02$ & $7.67-03$ & $2.95-02$ & $1.46-02$ & $5.92-03$ & $1.95-02$ & $1.09-02$ & 4.76-03 \\
\hline 3 & 10 & $5.47-03$ & 2.09-03 & 6.89-04 & $4.69-03$ & $1.78-03$ & 5.09-04 & $2.91-03$ & $1.29-03$ & $3.85-04$ \\
\hline 4 & 5 & $9.95-02$ & $5.61-02$ & $1.43-02$ & $8.19-02$ & $4.22-02$ & $8.52-03$ & $5.01-02$ & $2.70-02$ & $4.08-03$ \\
\hline 4 & 6 & $6.16-03$ & $1.96-03$ & 8.86-04 & $5.47-03$ & $1.73-03$ & $5.27-04$ & $3.51-03$ & $1.27-03$ & 2.33-04 \\
\hline 4 & 7 & $5.70-01$ & $5.66-01$ & $8.36-01$ & $6.24-01$ & $6.22-01$ & $9.37-01$ & $7.22-01$ & 7.13-01 & $1.07+00$ \\
\hline 4 & 8 & $1.66+00$ & $1.68+00$ & $2.45+00$ & $1.82+00$ & $1.85+00$ & $2.75+00$ & $2.11+00$ & $2.13+00$ & $3.13+00$ \\
\hline 4 & 9 & $8.62-02$ & $4.17-02$ & $4.67-02$ & $7.98-02$ & $3.70-02$ & $4.71-02$ & $6.48-02$ & $2.87-02$ & $4.98-02$ \\
\hline 4 & 10 & $1.08-02$ & $3.61-03$ & $1.24-03$ & $9.19-03$ & $3.03-03$ & $7.24-04$ & $5.56-03$ & $2.06-03$ & $3.18-04$ \\
\hline 5 & 6 & $9.36-03$ & $4.71-03$ & $4.20-03$ & $8.41-03$ & $4.01-03$ & $4.08-03$ & $6.46-03$ & $2.94-03$ & $4.16-03$ \\
\hline 5 & 7 & $2.29-02$ & $1.16-02$ & $4.32-03$ & $1.94-02$ & $9.45-03$ & $3.14-03$ & $1.25-02$ & $6.33-03$ & $2.31-03$ \\
\hline 5 & 8 & $6.64-02$ & $3.86-02$ & $4.81-02$ & $6.32-02$ & $3.47-02$ & $5.14-02$ & $5.70-02$ & $2.79-02$ & $5.62-02$ \\
\hline 5 & 9 & $2.42+00$ & $2.36+00$ & $3.61+00$ & $2.67+00$ & $2.59+00$ & $4.05+00$ & $3.13+00$ & $2.95+00$ & $4.58+00$ \\
\hline 5 & 10 & $7.82-01$ & 7.88-01 & $1.19+00$ & $8.62-01$ & 8.69-01 & $1.34+00$ & $1.01+00$ & $1.00+00$ & $1.52+00$ \\
\hline 6 & 7 & $6.39-02$ & $3.90-02$ & $1.46-02$ & $5.52-02$ & $3.29-02$ & $8.58-03$ & $3.59-02$ & $2.26-02$ & $3.76-03$ \\
\hline 6 & 8 & $4.27-02$ & $2.89-02$ & $2.44-02$ & $4.00-02$ & $2.80-02$ & $2.38-02$ & $3.32-02$ & $2.68-02$ & $2.34-02$ \\
\hline 6 & 9 & $2.99-02$ & $2.02-02$ & $6.61-03$ & $2.57-02$ & $1.68-02$ & $3.91-03$ & $1.64-02$ & $1.14-02$ & $1.84-03$ \\
\hline 6 & 10 & $6.92-03$ & $2.36-03$ & $8.21-04$ & $5.72-03$ & $1.84-03$ & $4.72-04$ & $3.36-03$ & $1.13-03$ & $2.15-04$ \\
\hline 7 & 8 & $1.76-01$ & $1.12-01$ & $7.11-02$ & $1.58-01$ & $1.04-01$ & $6.28-02$ & $1.18-01$ & $9.12-02$ & 5.66-02 \\
\hline 7 & 9 & $1.00-01$ & $6.45-02$ & $2.23-02$ & $8.64-02$ & $5.42-02$ & $1.35-02$ & $5.56-02$ & $3.70-02$ & $6.77-03$ \\
\hline 7 & 10 & $2.14-02$ & $7.48-03$ & $2.66-03$ & $1.76-02$ & $5.91-03$ & $1.50-03$ & $1.03-02$ & $3.59-03$ & $6.49-04$ \\
\hline 8 & 9 & $1.87-01$ & $1.16-01$ & $4.45-02$ & $1.61-01$ & $9.70-02$ & $2.86-02$ & $1.04-01$ & $6.76-02$ & $1.63-02$ \\
\hline 8 & 10 & $3.48-02$ & $1.30-02$ & $5.26-03$ & $2.86-02$ & $1.04-02$ & $3.30-03$ & $1.71-02$ & $6.54-03$ & $1.87-03$ \\
\hline 9 & 10 & $7.66-02$ & $6.00-02$ & $7.30-02$ & $7.69-02$ & $6.24-02$ & $7.73-02$ & $7.70-02$ & $6.71-02$ & $8.11-02$ \\
\hline
\end{tabular}

Notes. ${ }^{(a)}$ This work; ${ }^{(b)}$ Keenan (1988); ${ }^{(c)}$ Landi \& Bhatia (2008).

The corresponding effective collision strengths for the four transitions (see Fig. 5) are plotted in Fig. 6. For the optically forbidden transition $1-4$, our results are systematically higher than those of LB08 and K88 across the entire temperature range because of the clear resonance effects and a slightly higher background in the high-energy range. The resonance effects mainly enhance the effective collision strength for the optically allowed transition $1-13$ over the temperature range $10^{4} \mathrm{~K}-10^{5} \mathrm{~K}$. However, our effective collision strength for the optically allowed transition 2-7 agrees excellently with that of LB08. This is expected because of the weak resonance of the collision strength. For the spin-exchange transition 1-11, the resonance contribution is extremely high at lower temperatures, and the effective collision strengths converge to the values of LB08 at high temperatures.

The differences between our R-matrix effective collision strength and the results from LB08 for all transitions are also visually analysed in Fig. 7. We plot the ratios of effective collision strengths between our calculations and the results from LB08 for all 333 transitions available at three different temperatures $\left(\log _{10} T_{\mathrm{e}}(\mathrm{K})=6.23,6.53\right.$, and 6.93). One can see that our effective collision strengths are systematically higher than those of LB08, which can be explained by the resonance effects. There is generally a very good agreement between our collision strengths and the previous DW results. This agreement of $\Upsilon$ is better at higher temperatures (blue solid triangles) than at 
the lower temperatures (black solid squares), which is also expected from the behaviour of the collision strength due to the lack of resonances at higher incident electron energies. Table 4 contains a set of effective collision strengths for a sample of three electron temperatures for all transitions among the first ten finestructure levels arising from the $n=2$ complex of S XIII. In Table 4, We compare our DARC effective strengths with the results of K88 and LB08. As shown in Table 4, our effective collision strengths show an average difference of $29.6 \%, 23.6 \%$, and $14.3 \%$ at $\log _{10} T_{\mathrm{e}}(\mathrm{K}) 6.23,6.53$, and 6.93 , respectively, with the DW calculations of LB08. Overall, our effective collision strengths are consistent and closer at higher temperatures with the DW calculations due to the absence of significant contributions of resonances. The background of our collision strength agrees excellently with the results of LB08. By further considering the accuracy of $g f$-values involved in scattering calculations, we estimate our collision strength to be accurate to better than $20 \%$. For effective collision strengths, our results are estimated to be accurate to better than $15 \%$.

\section{Conclusions}

Electron-impact excitation data for Be-like sulfur were calculated with the DARC code among the 98 fine-structure levels (54 LS terms) belonging to the configurations $2 \mathrm{~s}^{x} 2 \mathrm{p}^{y}(x+y=2)$ and $2 \ln l^{\prime}\left(l=\mathrm{s}, \mathrm{p} ; n=3,4 ; l^{\prime}=\mathrm{s}, \mathrm{p}, \mathrm{d}\right.$ and $\left.\mathrm{f}\right)$. The target wavefunctions were generated by the GRASP0 code. Our energy levels, oscillator strengths, and transitions rates agree excellently with previous theoretical calculations and experimental measurements. The background values of our collision strength agree very well with previous DW calculations. The effective collision strengths were calculated over a temperature range $3.38 \times 10^{4} \mathrm{~K}-3.38 \times 10^{7} \mathrm{~K}$ important to astrophysically and plasma applications, and cover the whole equilibrium temperatures for Be-like sulfur. Regarding the effective collision strengths, we found strong differences with values available in the literature because these exclude resonances. The resonance contributions to the effective collision strength can be as high as several times (2-10) for forbidden transitions, in particular at low temperatures. However, the resonance contribution is not significant for most of the optically allowed transitions, especially at high temperatures. In conclusion, we provided a robust and complete set of R-matrix data of collision strengths and effective collision strengths for S XIII, which are useful for astrophysical and plasma applications.
Acknowledgements. The authors would like to thank C. P. Ballance for many fruitful discussions and valuable suggestions. This work was supported by the National Natural Science Foundation of China under grant Nos. 11273032, 11233004,11243004 , and 11103040 . Dr. G. Y. Liang acknowledges the support from the One-Hundred-Talents programme of the Chinese Academy of Sciences (CAS). Dr. M. A. Bari acknowledges the support by the Chinese Academy of Sciences Fellowship for Young International Scientists.

\section{References}

Acton, L. W., Bruner, M. E., Brown, W. A., et al. 1985, ApJ, 291, 865 Badnell, N. R. 1986, J. Phys. B Atom. Mol. Phys., 19, 3827

Ballance, C. P., \& Griffin, D. C. 2006, J. Phys. B Atom. Mol. Phys., 39, 3617 Bhattacharya, N., Bapat, B., Rangwala, S. A., Kumar, S. V. K., \& Krishnakumar, E. 1998, Eur. Phys. J. D, 2, 125

Bryans, P., Landi, E., \& Savin, D. W. 2009, ApJ, 691, 1540

Burgess, A., \& Sheorey, V. B. 1974, J. Phys. B Atom. Mol. Phys., 7, 2403

Du, S.-B., Yang, Z.-H., Chang, H.-W., \& Su, H. 2005, Chin. Phys. Lett., 22, 1638

Eissner, W., Jones, M., \& Nussbaumer, H. 1974, Comput. Phys. Commun., 8, 270

Feldman, U. 1992, Phys. Scr., 46, 202

Goldsmith, S., Oren, L., \& Cohen, L. 1974, ApJ, 188, 197

Grant, I. P., McKenzie, B. J., Norrington, P. H., Mayers, D. F., \& Pyper, N. C. 1980, Comput. Phys. Comm., 21, 207

Kavanagh, A. P., Watanabe, H., Li, Y. M., et al. 2010, Phys. Rev. A, 81, 022712

Keenan, F. P. 1988, Phys. Scr., 37, 57

Keenan, F. P., \& Berrington, K. A. 1985, Sol. Phys., 99, 25

Keenan, F. P., McCann, S. M., \& Widding, K. G. 1988, Sol. Phys., 117, 69

Khardi, S., Buchet-Poulizac, M. C., Buchet, J. P., et al. 1994, Phys. Scr., 49, 571

Landi, E., \& Bhatia, A. K. 2008, Atomic Data and Nuclear Data Tables, 94, 1

Landi, E., Doron, R., Feldman, U., \& Doschek, G. A. 2001, ApJ, 556, 912

Landi, E., Del Zanna, G., Young, P. R., Dere, K. P., \& Mason, H. E. 2012, ApJ, 744,99

Lepson, J. K., Beiersdorfer, P., Behar, E., \& Kahn, S. M. 2005, ApJ, 625, 1045

Li, F., Liang, G. Y., \& Zhao, G. 2013, ApJ, 762, 53

Mania, A. J., Luna, F. R. T., \& Hernandes, J. A. 2009, J. Quant. Spec. Radiat. Transf., 110, 82

Muehlethaler, H. P., \& Nussbaumer, H. 1976, A\&A, 48, 109

Norrington, P. H., \& Grant, I. P. 1987, J. Phys. B Atom. Mol. Phys., 20, 4869

Pegg, D. J., Forester, J. P., Vane, C. R., et al. 1977, Phys. Rev. A, 15, 1958

Raassen, A. J. J., Mewe, R., Audard, M., et al. 2002, A\&A, 389, 228

Sampson, D. H., Goett, S. J., \& Clark, R. E. H. 1984, Atomic Data and Nuclear Data Tables, 30, 125

Schippers, S., Bernhardt, D., Müller, A., et al. 2012, Phys. Rev. A, 85, 012513

Träbert, E., \& Heckmann, P. H. 1980, Phys. Scr., 22, 489

Tully, J. A., Seaton, M. J., \& Berrington, K. A. 1990, J. Phys. B Atom. Mol. Phys., 23, 3811

Yang, Z.-H., Du, S.-B., Chang, H.-W., et al. 2005, Chin. Phys. Lett., 22, 1099

Zhang, H. L., \& Fontes, C. J. 2013, Atomic Data and Nuclear Data Tables, 99, 416

Zhang, H. L., \& Sampson, D. H. 1992, Atomic Data and Nuclear Data Tables, 52,143 